\title{
Ultrasound/MRI Overlay with Image Warping for Neurosurgery
}

\author{
David G. Gobbi ${ }^{1}$, Roch M. Comeau ${ }^{2}$, and Terry M. Peters ${ }^{1,2}$ \\ 1 Imaging Research Laboratories, \\ John P. Robarts Research Institute, University of Western Ontario, \\ London ON N6A 5K8, Canada \\ dgobbi@irus.rri.on.ca, tpeters@irus.rri.on.ca \\ http://www.irus.rri.on.ca/Faculty/peters.html \\ 2 McConnell Brain Imaging Centre, Montreal Neurological Institute, \\ Montréal QC H3A 2B4, Canada \\ roch@nil.mni.mcgill.ca
}

\begin{abstract}
Performing a craniotomy will cause brain tissue to shift. As a result of the craniotomy, the accuracy of stereotactic localization techniques is reduced unless the brain shift can be accurately measured. If an ultrasound probe is tracked by a 3D optical tracking system, intraoperative ultrasound images acquired through the craniotomy can be compared to pre-operative MRI images to quantify the shift. We have developed 2D and 3D image overlay tools which allow interactive, realtime visualization of the shift as well as software that uses homologous landmarks between the ultrasound and MRI image volumes to create a thin-plate-spline warp transformation that provides a mapping between pre-operative imaging coordinates and the shifted intra-operative coordinages. Our techniques have been demonstrated on poly vinyl alcohol cryogel phantoms which exhibit mechanical and imaging properties similar to those of the human brain.
\end{abstract}

\section{Introduction}

\subsection{Stereotactic Surgery and Brain Shift}

Stereotactic surgery involves the registration of medical images, typically from volumetric modalities such as MRI and CT, with the head of the patient. With modern computer-aided surgical navigation equipment, the positions of surgical tools are tracked (usually by optical tracking systems) and the registration of the images to the head is accomplished with either bone-mounted or skin-mounted fiducial markers as follows: the markers are first identified in the image and their image coordinates are recorded, then the tracking-system coordinates of the fiducial markers are measured with a tracked pointer.

A basic assumption behind most of the applications of these 'frameless' stereotactic systems that the brain remains stationary during the surgery and that inaccuracies result only from registration and tracking errors. Following a 
craniotomy, however, brain shift in excess of $10 \mathrm{~mm}$ is not uncommon and the shift can be as large as $25 \mathrm{~mm}[1,2]$. This shift is a nonlinear warping of the brain caused by gravity, pressure inside the brain, and leakage of fluid from the cranial cavity. The extent of brain shift depends primarily on the size of the craniotomy and the duration of the surgery.

Brain shift can be significantly reduced by opening only a small drill-hole in the skull, as is typical of minimally-invasive stereotactic procedures. If stereotaxis is to be used after a craniotomy, however, the brain shift should be measured and corrected for to ensure sufficient positioning accuracy.

This paper discusses the use of intra-operative ultrasound imaging through the craniotomy as a method of quantifying the brain shift. A complementary project within our group is addressing this problem by tracking the cortical surface under the craniotomy $[3,4]$.

\subsection{Stereotactic Ultrasound Imaging}

A cluster of infrared LEDs attached to the biopsy needle mount of our ultrasound probe allows the surgical workstation to track the position and orientation of the probe in real-time as the ultrasound video image is captured. Via the tracking system, the slice of patient anatomy that matches the ultrasound video image can be determined. The corresponding oblique slice can be extracted from the pre-operative MRI image volume for comparison. This basic technique has been demonstrated by several groups $[5,6,7,8]$. Our previous contributions include a comprehensive study of this technique involving both phantom work and several clinical procedures $[9,10,11]$. At least one company, Sofamor Danek, supports this technique through their SonoNav product (www.sofamordanek.com/sono.htm).

The primary goal of our current study is twofold. First, we are investigating better interactive methods for visualizing the ultrasound video and the corresponding MR slices, in order to provide surgeons or radiologists with tools for very rapid semi-quantitative assessment of brain shift. Second, we are aiming towards rapid and accurate quantification of the brain shift in order to provide a nonlinear warp transformation from pre-operative image space to intra-operative image space. For interactive assessment of brain shift, 2D ultrasound video is used. For quantitative measurement of brain shift over a substantial volume of brain tissue, 3D ultrasound imaging is more suitable.

The creation of the nonlinear warp transformation is accomplished through the identification of several homologous landmarks in the ultrasound image volume and the MR image volume. The warp transformation is interpolated between the landmarks through the use of a radial basis function interpolation technique. We are currently using thin plate splines [12] but are investigating other methods of interpolation (including those which incorporate a mechanical model of brain tissue). 


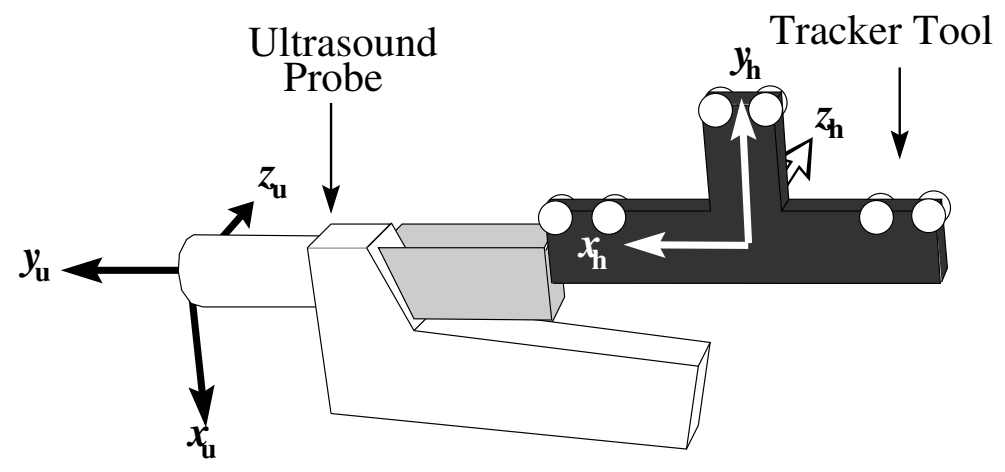

Fig. 1. A POLARIS tracker tool with infrared LEDs is mounted on the ultrasound probe via its biopsy needle mount.

\section{Materials and Methods}

The primary components of our system are Aloka SSD-2000 and SSD-1700 ultrasound scanners with a $5 \mathrm{MHz}$ burrhole ultrasound probe (Aloka Co., Ltd, Tokyo 181-8522, Japan), a POLARIS optical tracking system (Northern Digital Inc., Waterloo ON N2V 1C5, Canada, www.ndigital.com), and a $450 \mathrm{MHz}$ Pentium II workstation with video input/output capabilities.

A set of infrared LEDs are mounted on the ultrasound probe as shown in Figure 1. The respective coordinate systems of the ultrasound image and of the POLARIS LEDs are labelled in the figure. The coordinate transformation between the ultrasound image and the tracking system was determined through a calibration procedure which we have described in the past [13]. A similar method is used by another group [14], and a set of very different but more widely used methods are described in the literature with reference to freehand $3 \mathrm{D}$ ultrasound reconstruction [15].

Our visualization methods and the overall image registration were tested using a special-purpose poly (vinyl alchohol) cryogel (PVA-C) phantom $[17,18]$. This phantom is described in detail in [11].

Our software has been developed using the open-source Visualization Toolkit, VTK (http://www.kitware.com/vtk.html) [16]. Several C++ classes which were written for this project have been contributed to the VTK class library. The first, vtkImageReslice, efficently resamples an image based on a linear, perspective, or nonlinear warp transformation. The second, vtkVideoSource, provides a VTK interface for any video digitizer which is compatible with either the Matrox Imaging Library or Microsoft Video for Windows. The third, vtkImageBlend, provides transparency blending of images. We have also contributed significant optimizations for image display in VTK.

Our most significant recent contribution to VTK is a new class heirarchy for geometrical transformations. Two of the several new geometrical transformation classes were developed specifically for this project: the vtkThinPlateSpline- 
Transform class provides a landmark-based warp transformation using either a thin-plate spline radial basis function $(\mathrm{RBF})$ or any arbitrary RBF, while the vtkGridTransform defines a warp transformation via a grid of displacement vectors. The tight integration of these classes with VTK provides the following: 1) scalability, in that the image warping in VTK will automatically parallelize over all processors on a multi-processor platform, 2) generality, in that the transformations can be applied to images, vertices, vector fields, or implicit functions and 3) flexibility, in that concatenation and inversion of arbitrary transformations is supported.

In addition to those classes which we have contributed to VTK, we have also developed classes that interface with the POLARIS optical tracking system and which perform 3D ultrasound reconstruction. We have also written a large set of foundation classes for rapid development of visualization applications in the Python programming language. These foundation classes and several example applications will be made available under an open-source license from http://www.atamai.com.

\subsection{Image Overlay}

Our system provides the means of overlaying both 2D ultrasound video and $3 \mathrm{D}$ ultrasound image volumes with 3D MRI volumes. The 2D video overlay is interactive, providing refresh rate of 10 frames per second, and is designed to allow the surgeon or neuroradiologist to rapidly make a semi-quantitative assessment of the brain shift. The 3D overlay is for use in tagging homologous landmarks between a 3D ultrasound volume and an MRI volume. Once a suitable set of landmarks have been identified, a nonlinear warp transformation from preoperative MRI image coordinates to intra-operative surgical coordinates can be generated. The 3D overlay is discussed more fully in the next section.

A video overlay session with a PVA-C phantom is shown in Figure 2. The top window is a rendering of three orthogonal slices through the MRI volume, as well as a virtual model of the ultrasound probe which moves in real-time as the "real" optically-tracked ultrasound probe is moved. The virtual ultrasound probe includes an overlay of the ultrasound video (shown in orange) on the corresponding MRI slice (shown in grey). The 3D scene can be interactively reoriented (rotate, pan, zoom) with the mouse.

A strictly $2 \mathrm{D}$ view of the ultrasound/MR overlay is shown in the bottom-left window. This view provides the ultrasound operator with a means of interactively evaluating the brain shift. The ultrasound video is displayed with an orange color scale to provide good contrast with the greyscale MRI. A slider below this window allows the user to control the opacity of the ultrasound overlay.

The raw ultrasound video is also displayed (in the bottom-right window) so that the ultrasound operator will never have a need to divide his or her attention between the computer monitor and the ultrasound machine's CRT display. 


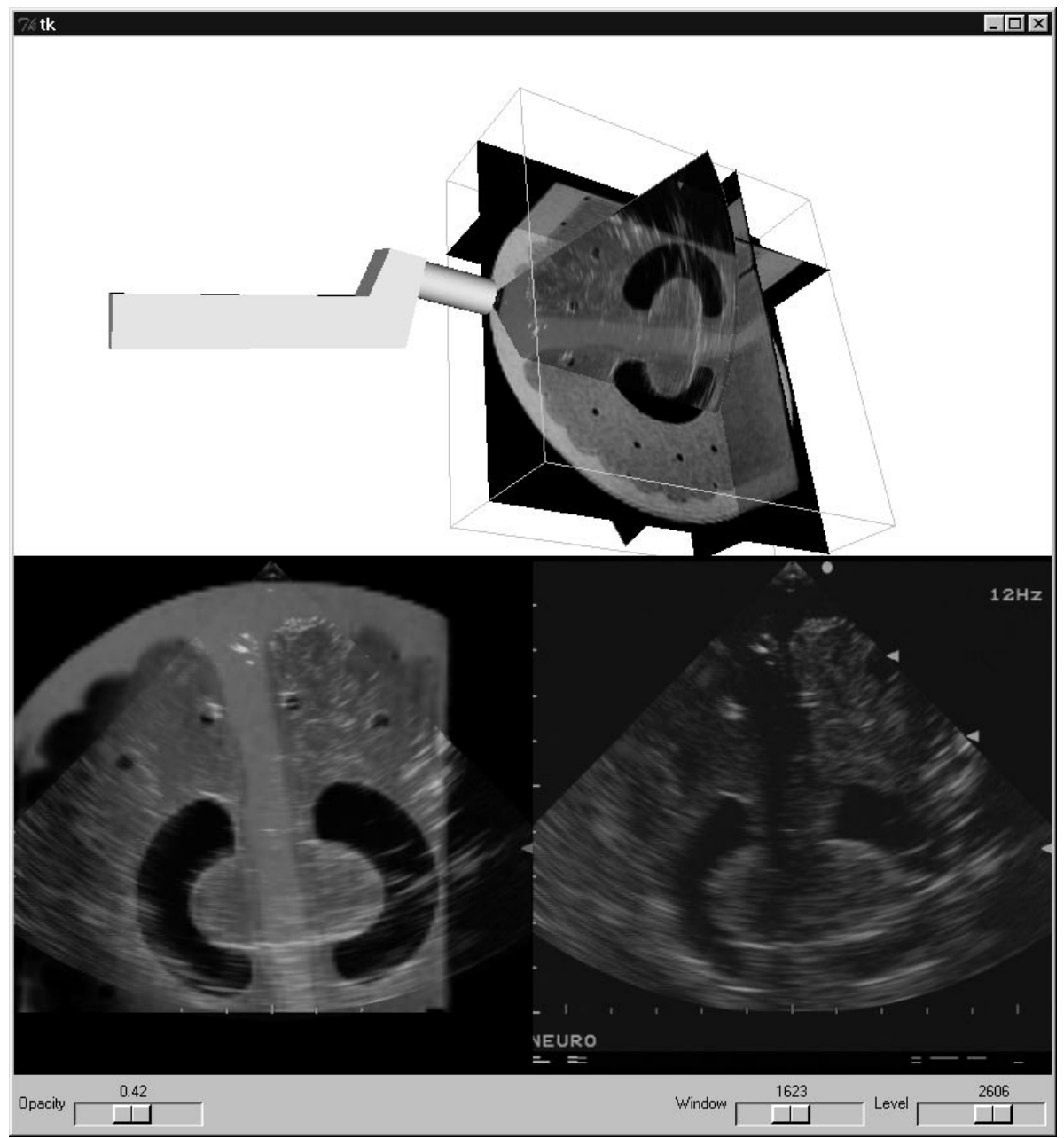

Fig. 2. Overlay of ultrasound video on an MRI volume. These data were obtained from one of our deformable PVA-C phantoms. The rendering frame rate is approximately 10 frames/second.

\section{$3 \quad$ Image Warping}

We use a set of homologous landmarks in the ultrasound image volume and the MRI volume to describe the nonlinear warp transformation from pre-operative MRI image space to intra-operative ultrasound image space. Currently, thin plate spline interpolation of the warp transformation is performed between the landmark points. In the future other interpolation methods will be investigated. 
The pre-operative image is warped, along with any tags or labels on the image, into the intra-operative image space.

The landmarks are identified on a 3D Ultrasound/3D MRI overlay as shown in Figure 3. Figure 3(a) is a volume rendering of the MRI of the phantom, provided to indicate the geometry of the phantom. Figure 3(b) is an MRI volume fused with a $3 \mathrm{D}$ ultrasound volume (in orange) from a tracked ultrasound probe. The registration of the volumes was achieved entirely via the tracking of the probe by the POLARIS. The misregistration between the ultrasound and MRI at the bottom of the bean-shaped cavities (which simulate the ventricles in the brain) is due to speed of sound differences between the PVA-C and water. In the future, we will experimentally determine the correct mixture of glycerol and water to obtain the same speed of sound in the cavities as in the phantom material.

To simulate brain shift, we applied a warp to both images as shown by the arrow in Figure 3(c). This simulated warp provides a "gold standard" to which a landmark-driven warp (Figure 3(d)) can be compared.

We used the following method to create the landmark driven warp: Triplanar views of the warped 3D ultrasound volume and non-warped MRI volume were placed side by side. Corresponding landmarks were tagged in the ultrasound and MRI - the landmarks which were used are the small circles which form a ring around the ventricular cavities in the centre of the phantom. The small circles represent large cerebral blood vessels which could be used as landmarks in a real brain. Only the top eight circlular landmarks were used because the others were not within the volume covered by the ultrasound scan.

The landmark coordinates were used to accomplish a thin plate spline warp transformation of the MRI image to match it to the ultrasound image. Comparing Figure 3(c) and (d), the warp transformation matches the simulated ventricles in the MRI images very well. The indentation on the right side of the surface of the phantom is not duplicated, however, because it lies well outside of the cluster of landmarks used for nonlinear registration.

\section{Conclusion}

Early results of using homologous landmarks to nonlinearly warp an MRI image volume to match a 3D ultrasound volume suggest that good accuracy in the warp is possible as long as the landmarks provide sufficient coverage of the target region of interest within the volumes. In terms of the use of this methodology on patients, this means that landmarks must be chosen which lie between the craniotomy and the region of interest, and if possible should surround the region of interest. The ultimate goal is to match the morphology of the pre-operative MRI volume to the intra-operative morphology of brain as demonstrated by $3 \mathrm{D}$ ultrasound, using the landmark-based warp scheme described above.

Our support for live video overlay is full-featured and provides an interactive frame rate ( $>10$ frames per second). We aim to further improve the overlay by adding the ability freeze and then annotate the video image. Another important 
(a)

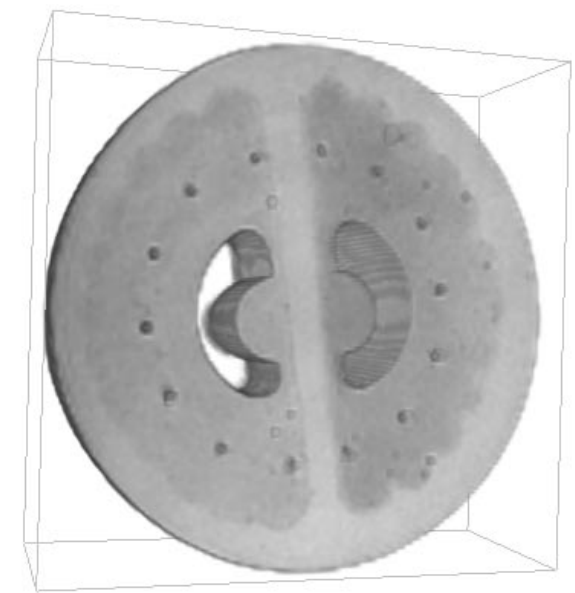

(c)

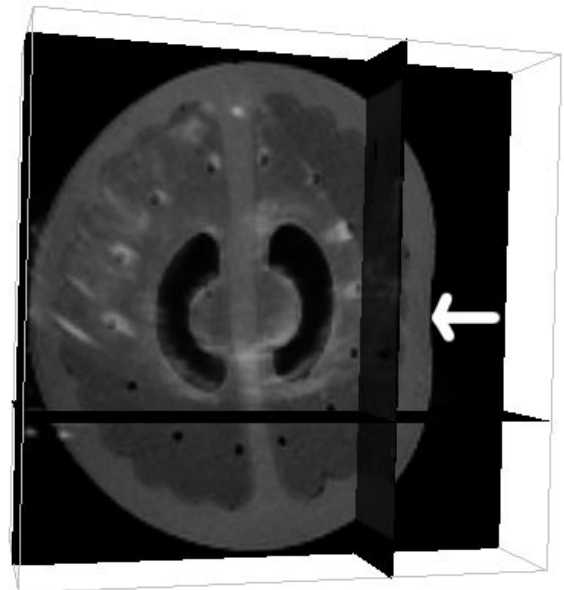

(b)

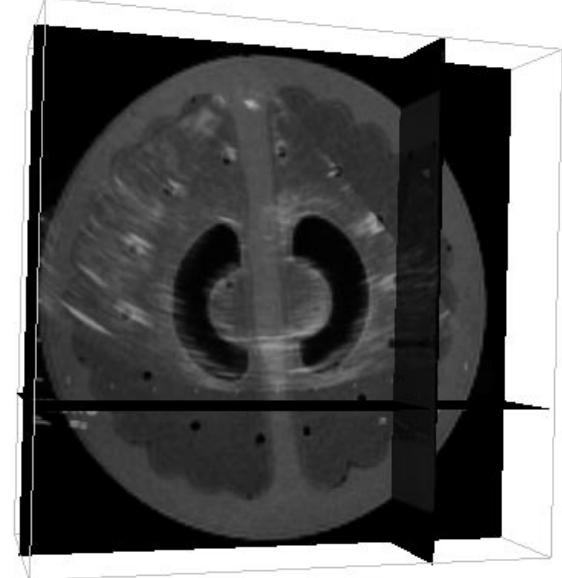

(d)

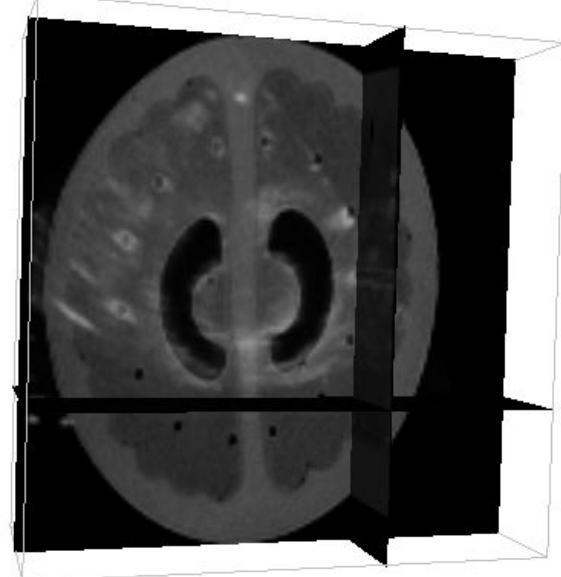

Fig. 3. Orthogonal slice planes through a $3 \mathrm{D}$ overlay of ultrasound and $\mathrm{MR}$ image volumes. 
area for future work will be the development of a surgeon-friendly user interface (e.g. by mounting buttons on the ultrasound probe to control the opacity of the overlay or the window/level of the MRI), to make the system as suitable as possible as a routine operating-room tool.

\section{Acknowledgments}

PVA-cryogel phantoms used for this project were constructed by Kathleen Surry at Robarts, and our surgical navigation system was developed as a shared effort between the first author and Dr. Yves Starreveld. Funding for this research is provided through grants from the Medical Research Council of Canada and the Canadian Foundation for Innovation. Scholarship funding for David Gobbi is provided by the Ontario Ministry of Education and by the University of Western Ontario.

\section{References}

1. D. L. G. Hill, C. R. Maurer Jr., R. J. Maciunas, J. A. Barwise, J. M. Fitzpatrick and M.Y. Wang. Measurement of Intraoperative Brain Surface Deformation under a Craniotomy. Neurosurgery 43:514-528, 1998.

2. D. W. Roberts, A. Hartov, F. E. Kennedy, M. I. Miga and K. D. Paulsen. Intraoperative Brain Shift and Deformation: A Quantitative Analysis of Cortical Displacement in 28 Cases. Neurosurgery 43:749-760, 1998.

3. M.A. Audette and T.M. Peters. Level-Set Segmentation and Registration for Computing Intrasurgical Deformations. Proc. SPIE Medical Imaging 99, SPIE Vol. 3661:110-121, 1999

4. M.A. Audette, K. Siddiqi and T.M. Peters. Level-set Surface Segmentation and Fast Cortical Range Image Tracking for Computing Intrasurgical Deformations. Medical Image Computing and Computer Assisted Intervention - MICCAI'99. C. Taylor and A. Cholchester (eds) Lecture Notes in Computer Science 1679:788-797, SpringerVerlag, Berlin, 1999

5. R .D. Bucholz, D. Yeh,, J. Trobaugh, L. L. McDurmont, C. D. Sturm, C. Baumann and M. H. Jaimie. The Correction of Stereotactic Inaccuracy Caused by Brain Shift Using an Intraoperative Ultrasound Device. CVRMed-MRCAS '97 : First Joint Conference Computer Vision, Virtual Reality and Robotics in Medicine and Medical Robotics and Computer-Assisted Surgery, Troccaz, J., Grimson, E., Mösges, R., (eds.), Grenoble, France, Springer-Verlag, Berlin, 1997.

6. C. Giorgi and D.S. Casolino. Preliminary Clinical Experience with Intraoperative Stereotactic Ultrasound Imaging. Stereotactic and Functional Neurosurgery 68:5458, 1997.

7. N. Hata, T. Dohi, H. Iseki and K. Takakura. Development of a Frameless and Armless Stereotactic Neuronavigation System with Ultrasonographic Registration. Neurosurgery 41:608-614, 1997.

8. A. Jödicke, W. Deinsberger, H. Erbe, A. Kriete and D.-K. Böker. Intraoperative Three-Dimensional Ultrasonography: An Approach to Register Brain Shift Using Multidimensional Image Processing. Minim. Invasive Neurosurg. 41:13-19, 1998. 
9. R.M. Comeau, A. Fenster and T.M. Peters. Integrated MR and Ultrasound Imaging for Improved Guidance in Neurosurgery. Proc SPIE, Medical Imaging '98, SPIE Vol. 3338:474-754, 1998.

10. R.M. Comeau, A. Fenster and T.M. Peters. Intraoperative US in Interactive ImageGuided Neurosurgery. Radiographics 18:1019-1027, 1998.

11. R.M. Comeau, A.F. Sadikot, A. Fenster and T.M. Peters. Intraoperative Ultrasound for Guidance and Tissue Shift Correction in Image-Guided Surgery. Medical Physics 27:787-800, 2000.

12. F.L. Bookstein. Linear Methods for Nonlinear Maps. In Brain Warping, A.W. Toga (ed). Academic Press, San Diego, 1999.

13. D.G. Gobbi, R.M. Comeau and T.M Peters. Ultrasound Probe Tracking for RealTime Ultrasound/MRI Overlay and Visualization of Brain Shift. Medical Image Computing and Computer Assisted Intervention - MICCAI'99. C. Taylor and A. Cholchester (eds) Lecture Notes in Computer Science 1679:920-927, SpringerVerlag, Berlin, 1999.

14. N. Pagoulatos, W.S. Edwards, D.R. Haynor and Y. Kim. Interactive 3-D Registration of Ultrasound and Magnetic Resonance Images Based on a Magnetic Position Sensor. IEEE Trans. Inf. Tech. in Biomed. 3:278-288, 1999.

15. R.W. Prager, R.N. Rohling, A.H. Gee and L. Berman. Rapid Calibration for 3-D Freehand Ultrasound. Ultrasound in Med. Biol. 24:855-868, 1998.

16. W. Schroeder, K. W. Martin and W. Lorensen. The Visualization Toolkit, 2nd Edition. Prentice Hall, Toronto, 1998.

17. I. Mano, H. Goshima, M. Nambu and I. Masahiro. New Polyvinyl Alcohol Gel Material for MRI Phantoms. Magnetic Resonance in Medicine 3: 921-926, 1986.

18. K. Chu and B. Rutt. Polyvinyl Alcohol Cryogel: An Ideal Phantom Material for MR Studies of Arterial Flow and Elasticity. Magnetic Resonance in Medicine 37:314319, 1997. 